Applied Physics B manuscript No.

(will be inserted by the editor)

\title{
Spectral interferometry and reflectometry used to measure thin films
}

\author{
P. Hlubina, J. Luňáček, D. Ciprian, R. Chlebus \\ Department of Physics, Technical University Ostrava, 17. listopadu 15, 70833 Ostrava-Poruba, Czech Republic
}

Received:

\begin{abstract}
A new method for a precise measurement of the oscillatory part of phase change on reflection (interferometric phase) from a thin-film structure is presented. The method, which is based on phase retrieval from the spectral interferograms recorded at the output of a slightly dispersive Michelson interferometer, is combined with reflectometry. The interferometric phase of the thin-film structure is measured precisely using a reference sample of known phase change on reflection. The spectral reflectance of the thin-film structure is also measured in the interferometer. The feasibility of the method is confirmed in processing the experimental data for $\mathrm{SiO}_{2}$ thin film on a silicon wafer of known optical constants. Four samples of the thin film are used and their thicknesses are determined. We confirm very good agreement between the thicknesses obtained from the interferometric phase and reflectance measurements.
\end{abstract}

PACS 07.60.Ly, 68.55.Jk, 78.20

\section{Introduction}

Optical methods, based on ellipsometric $[1,2]$, reflectometric [3] or interferometric [4-6] measurements, belong to the most important ones in determining the parameters and characteristics of thin-film structures. Ellipsometric measurements performed at a single wavelength and a fixed angle of incidence provide the film thickness and optical constants [1]. Measurements by spectroscopic ellipsometry provide the results over a wide wavelength range with greater precision and accuracy [2]. Normal incidence spectroscopic reflectometry [3] applied over a wide wavelength range is a useful tool for the characterization of thin films and multilayer structures

Correspondence to: P. Hlubina, Phone: 420597323 134,

Fax: 420597323 403, E-mail: petr.hlubina@vsb.cz commonly encountered in semiconductor industry. Accurate and precise characterization of many structures that cannot be measured with either technique alone or with combined reflectometry and single wavelength ellipsometry is possible by employing sophisticated parametric dispersion models and simultaneously fitting both the ellipsometric and reflectance spectra.

The optical method most commonly employed for micrometer-scale thickness measurements is Fourier transform infrared [4] and white-light [5] interferometry. The use of white-light interferometry was extended into the spectral domain $[6,7]$ where the phase of the reflected wave, which changes as a function of wavelength [8] and layer thickness, is inscribed in the recorded spectral interferogram.

Recently, we used dispersive white-light spectral interferometry for measuring the thickness of $\mathrm{SiO}_{2}$ thin film on a silicon wafer [10]. The technique utilizes a slightly dispersive Michelson interferometer with one of the mirrors replaced by the thin-film structure of known optical constants. The thickness of the thin film is determined from the fit of the recorded spectral interferogram to the theoretical one. More recently, the use of dispersive white-light spectral interferometry was extended for measuring thin film thickness utilizing the absolute phase retrieval from the spectral interferogram $[11,12]$. However, the results suffer from the systematic phase errors due to the optical components present in the interferometer. To minimize them, a procedure with the reference measurement needs to be applied [7].

In this paper, we present a new method for a precise measurement of the interferometric phase (nonlinearlike phase $[11,12]$ ) of a thin-film structure. The method is based on white-light spectral interferometry and is combined with reflectometry. The nonlinear-like phase is measured in a slightly dispersive Michelson interferometer and a reference sample of known phase change on reflection is used to compensate the phase errors introduced by the optical components of the interferometer. In the same interferometer, the spectral reflectance 


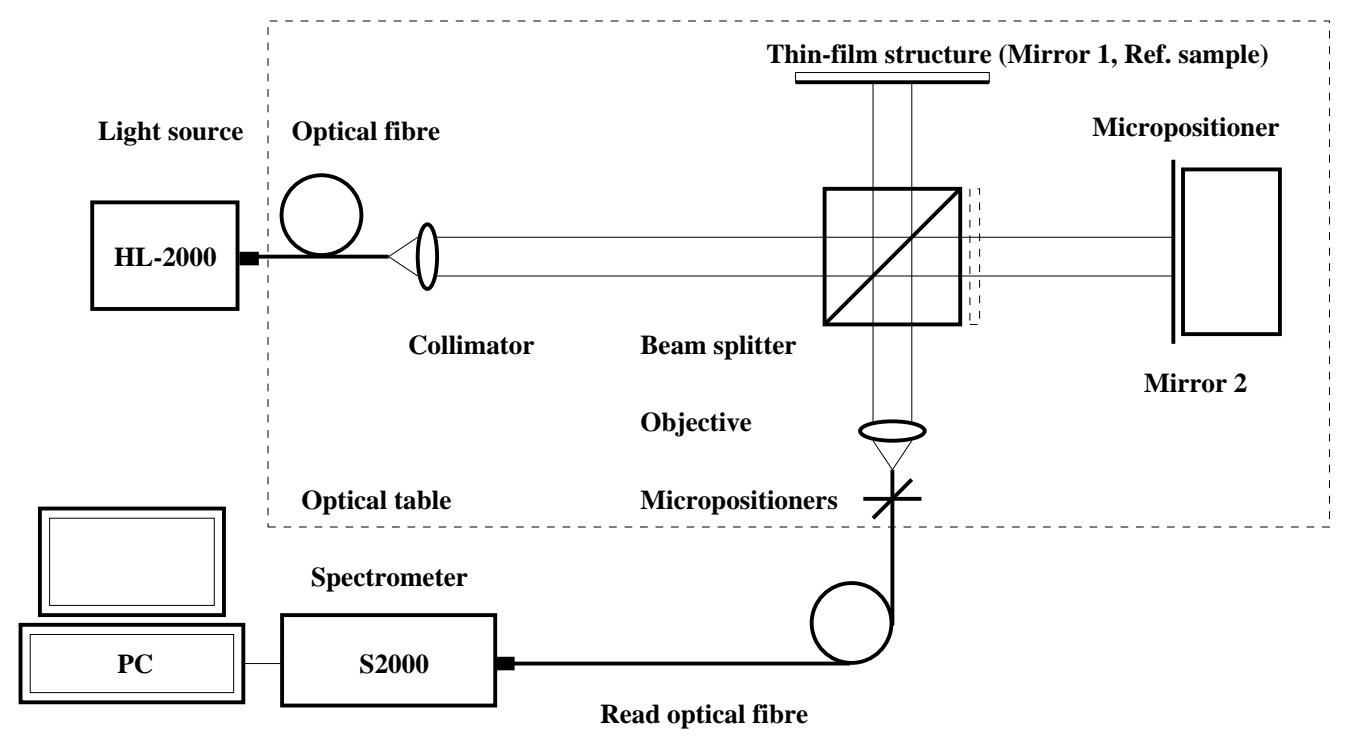

Fig. 1 Experimental set-up with a Michelson interferometer to measure the nonlinear-like phase and reflectance of a thin-film structure.

of the thin-film structure is measured. We show that the thickness of $\mathrm{SiO}_{2}$ thin film on a silicon wafer determined from the precisely retrieved nonlinear-like phase agrees well with that determined from the measured reflectance.

\section{Theoretical background}

Let us consider the mutual interference of two beams from a broadband source at the output of a Michelson interferometer (see Fig. 1) with a cube beam splitter of the effective thickness $t_{\text {eff }}[10]$ and metallic mirrors 1,2 that are characterized by complex reflection coefficients

$$
r_{j}(\lambda)=\sqrt{R_{j}(\lambda)} \exp \left[\mathrm{i} \delta_{j}(\lambda)\right]
$$

where $R_{j}(\lambda)$ and $\delta_{j}(\lambda)$, respectively, are the wavelengthdependent mirror reflectances and phase changes on reflection from the mirrors $(j=1,2)$. We assume that the geometrical path lengths of the light rays in dispersive glass of the beam splitter are not the same for both interferometer arms and the beam splitter has to be represented by an ideal beam splitter and a plate of the same dispersion (see a dashed element in Fig. 1) and of the thickness $t_{\text {eff }}$. The Michelson interferometer is referred to as slightly dispersive $[10,11]$ and the optical path difference (OPD) between the beams is given by

$\Delta(\lambda)=2 L+2 n(\lambda) t_{\mathrm{eff}}-\lambda\left[\delta_{1}(\lambda)-\delta_{B S}(\lambda)-\delta_{2}(\lambda)\right] /(2 \pi)$,

where $2 L$ is the difference of path lengths between the interfering beams in the air whose dispersion is neglected, $n(\lambda)$ is the refractive index of the beam splitter material and $\delta_{B S}(\lambda)$ is the phase change at the output of the interferometer due to the beam splitter. If the used mirrors are identical, i. e. $\delta_{1}(\lambda)=\delta_{2}(\lambda)$, only the effect of the phase change $\delta_{B S}(\lambda)$ is inscribed in the OPD $\Delta(\lambda)$. Moreover, some commercially available beam splitters (cubic ones) are with $\delta_{B S}(\lambda)=0$ so that their effective thicknesses $t_{\text {eff }}$ can be measured precisely [13].

Next, let us consider that mirror 1 of the interferometer is replaced by a thin-film structure, which is characterized by a complex reflection coefficient

$$
r(\lambda)=\sqrt{R(\lambda)} \exp \left[\mathrm{i} \delta_{r}(\lambda)\right]
$$

where $R(\lambda)$ and $\delta_{r}(\lambda)$, respectively, are the reflectance and the phase change on reflection. The OPD between beams in the interferometer is given by

$\Delta(\lambda)=2 L+2 n(\lambda) t_{\mathrm{eff}}-\lambda\left[\delta_{r}(\lambda)-\delta_{B S}(\lambda)-\delta_{2}(\lambda)\right] /(2 \pi)$.

When light is incident on a surface of a thin-film structure (a uniform thin film on a substrate), multiple reflections take place and the complex reflection coefficient can be expressed according to the well-known relations [1]. The phase change $\beta(\lambda)$ that the reflected wave experiences as it traverses the thin film once from one boundary to the other is given at normal incidence by

$$
\beta(\lambda)=\frac{2 \pi}{\lambda} n_{1}(\lambda) d,
$$

where $n_{1}(\lambda)$ is the refractive index of the thin film and $d$ is its thickness. The phase change $\delta_{r}(\lambda)$ can be represented as the sum of two contributions

$$
\delta_{r}(\lambda)=2 \beta(\lambda)+\phi_{n l}(\lambda)
$$

where $\phi_{n l}(\lambda)$ is the nonlinear phase function due to the multiple reflections within the thin film $[5,6]$. As an example, Figs. 2 and 3 show the nonlinear phase function $\phi_{n l}(\lambda)$ and the reflectance $R(\lambda)$ of $\mathrm{SiO}_{2}$ thin film 


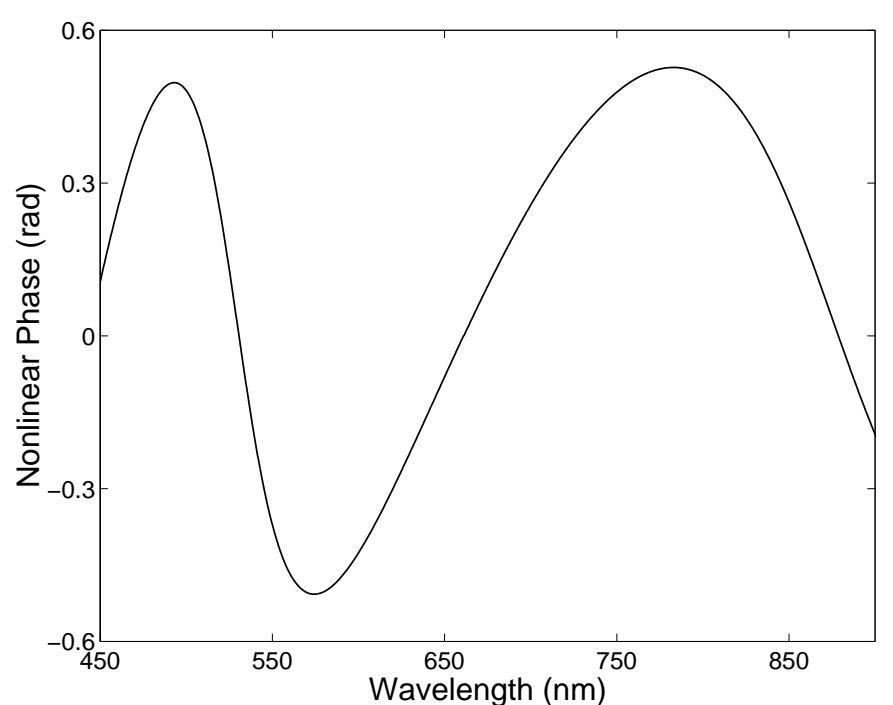

Fig. 2 Theoretical nonlinear phase of $\mathrm{SiO}_{2}$ thin film of thickness $d=450 \mathrm{~nm}$ on the silicon substrate.

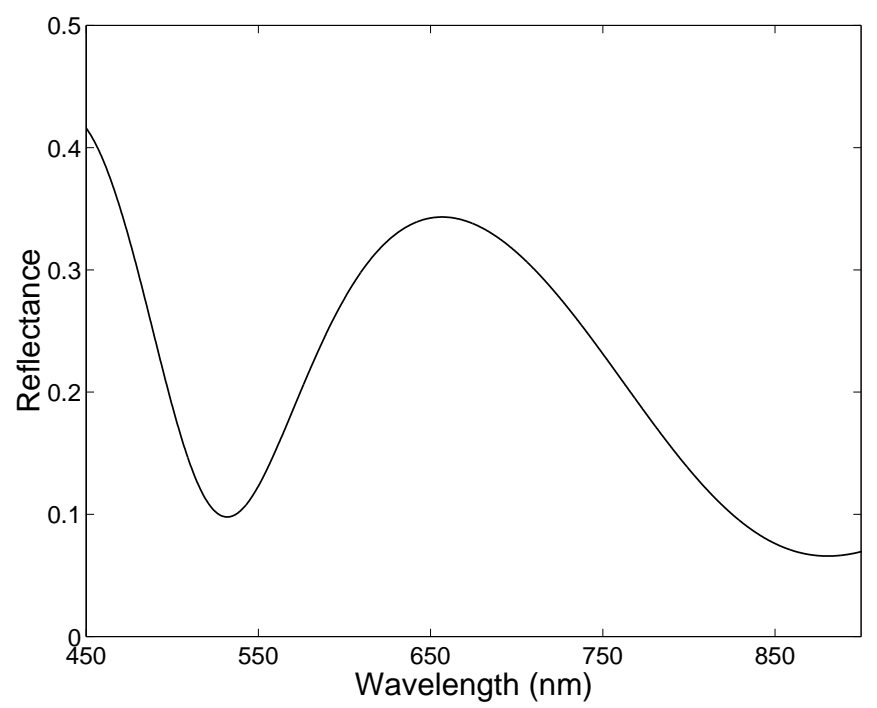

Fig. 3 Theoretical reflectance of $\mathrm{SiO}_{2}$ thin film of thickness $d=450 \mathrm{~nm}$ on the silicon substrate.

of thickness $d=450 \mathrm{~nm}$ on a silicon substrate. The quantities were computed from the complex reflection coefficient of the structure $[1,10]$ which takes into account the known wavelength dependences for the refractive index $n_{1}(\lambda)$ of the $\mathrm{SiO}_{2}$ thin film and the complex refractive index of the silicon substrate [14].

We showed $[11,12]$ that the oscillatory part of phase change on reflection (interferometric phase) from the thin-film structure, which is referred to the nonlinearlike phase function and which is similar to the nonlinear phase function $\phi_{n l}(\lambda)$, can be retrieved from the spectral interferograms recorded in the experimental set-up shown in Fig. 1. In the same set-up the reflectance measurements can be performed.

\section{Experimental methods}

We propose new procedures for measurement of both the the nonlinear-like phase function and reflectance of a thin-film structure in the same experimental set-up.

\subsection{Method of spectral interferometry}

It results from previous papers $[11,12]$ that the spectral interferograms recorded in the mentioned experimental set-up can be used to measure the absolute OPD $\Delta(\lambda)$, from which we can construct the nonlinear-like phase function $\delta(\lambda)$ for a chosen mirror position $L=L_{0}$. Under the assumption $\delta_{B S}(\lambda)=0 \mathrm{Eq}$. (4) gives the relation

$$
\delta(\lambda)=(2 \pi / \lambda)\left[2 L_{0}+2 n(\lambda) t_{\mathrm{eff}}-\Delta(\lambda)\right]+\delta_{2}(\lambda),
$$

where $\delta_{2}(\lambda)$ is the phase change on reflection from mirror 2 . To compensate the phase change $\delta_{2}(\lambda)$, we propose a next measurement step with the reference sample used instead of mirror 1 . In this case we measure the OPD $\Delta_{\text {ref }}(\lambda)$ and for the chosen mirror position $L_{0}$ and for the reference phase change on reflection $\delta_{\text {ref }}(\lambda)$, which can be computed, we obtain

$$
\delta_{2}(\lambda)=\delta_{\text {ref }}(\lambda)-(2 \pi / \lambda)\left[2 L_{0}+2 n(\lambda) t_{\text {eff }}-\Delta_{\text {ref }}(\lambda)\right]
$$

It should be stressed here that the phase change $\delta_{2}(\lambda)$ given by Eq. (8) is not determined absolutely (it can be shifted by a constant value).

\subsection{Method of spectral reflectometry}

The procedure for the reflectance measurement in the experimental set-up is with the blocked arm of mirror 2 and it consists of three steps: first by blocking the source, the background spectrum $I_{b k g}(\lambda)$ is measured, second by using a reference sample instead of mirror 1 , the reference reflection spectrum $I_{r e f}(\lambda)$ is measured, and third by using the thin-film structure instead of the reference sample, the reflection spectrum $I_{\text {meas }}(\lambda)$ of the thin-film structure is measured. The absolute reflectance $R(\lambda)$ of the thin-film structure is given by

$$
R(\lambda)=\frac{I_{\text {meas }}(\lambda)-I_{b k g}(\lambda)}{I_{r e f}(\lambda)-I_{b k g}(\lambda)} R_{r e f}(\lambda),
$$

where $R_{r e f}(\lambda)$ is the theoretical reflectance of the reference sample. It should be noted here that the spectra in both the nominator and denominator of Eq. (9) are affected by a lot of factors such as the spectral transmittance of the beam splitter and the spectral sensitivity of the spectrometer, but we eliminate them by the above procedure. 


\section{Experimental set-up}

The experimental set-up used in the application of spectral interferometry and reflectometry to measure thin films is shown in Fig. 1. It consists of a white-light source: a halogen lamp HL-2000 (Ocean Optics, Inc.) with launching optics, an optical fibre and a collimating lens, a bulkoptic Michelson interferometer with a cube beam splitter made of BK7 optical glass, a metallic mirror connected to a micropositioner, a thin-film structure, a microscope objective, micropositioners, a read optical fibre, a miniature fibre-optic spectrometer S2000 (Ocean optics, Inc.), an A/D converter and a personal computer.

The thin-film structure is represented by a uniform $\mathrm{SiO}_{2}$ thin film on the silicon wafer. Four different samples with four different $\mathrm{SiO}_{2}$ thin-film thicknesses were under study. The $\mathrm{SiO}_{2}$ thin films on the silicon wafers were prepared using a dry oxidation process described by the so-called Deal-Grove model [15]. Single-crystal silicon wafers from ON Semiconductor, Czech Republic, were characterized by subsequent parameters: diameter $(100 \pm 0.5) \mathrm{mm}$, orientation (111), B doped type P, thickness $(381 \pm 25) \mu \mathrm{m}$ and resistivity $(0.008 \div 0.009) \Omega \mathrm{cm}$. Before the oxidation, the wafers were cut into $40 \times 40 \mathrm{~mm}$ squares, cleaned by standard methods and then annealed in a furnace at $1200^{\circ} \mathrm{C}$. According to the model, four annealing times were selected in order to prepare $\mathrm{SiO}_{2}$ thin film of four different thicknesses ranging approximately from 300 to $450 \mathrm{~nm}$.

\section{Experimental results and discussion}

First, the effective thickness $t_{\text {eff }}$ of the beam splitter made of BK7 glass was determined by a procedure presented in a previous paper [13]. We used two identical mirrors in the experimental set-up and confirmed from the linear dependence of the measured OPD $\Delta(\lambda)$ on the refractive index $n(\lambda)$ of BK7 glass that the phase change $\delta_{B S}(\lambda) \approx 0$. Prior to the measurement of the nonlinear-like phase $\delta(\lambda)$ of the thin-film structure, the phase change $\delta_{2}(\lambda)$ needs to be determined. We used the procedure presented above with a silicon wafer as the reference sample. Figure 4 shows the corresponding phase function $\delta_{2}(\lambda)$ which was determined from the measured OPD $\Delta_{r e f}(\lambda)$ by using Eq. (8) when the phase change $\delta_{\text {ref }}(\lambda)$ on reflection from the silicon wafer was computed using the data available from literature [14]. Figure 4 clearly demonstrates decreasing phase with increasing wavelength which is much steeper in comparison with that for aluminium [9] as shown by the dashed line in Fig. 4. This is due to protective coating of the mirror.

Next, the OPD $\Delta(\lambda)$ was measured for the thin-film structure and the known phase function $\delta_{2}(\lambda)$ was used in Eq. (7) for precise determination of the nonlinear-like phase $\delta(\lambda)$. The function was compared with theory in order to determine the thickness $d$ of the first sample

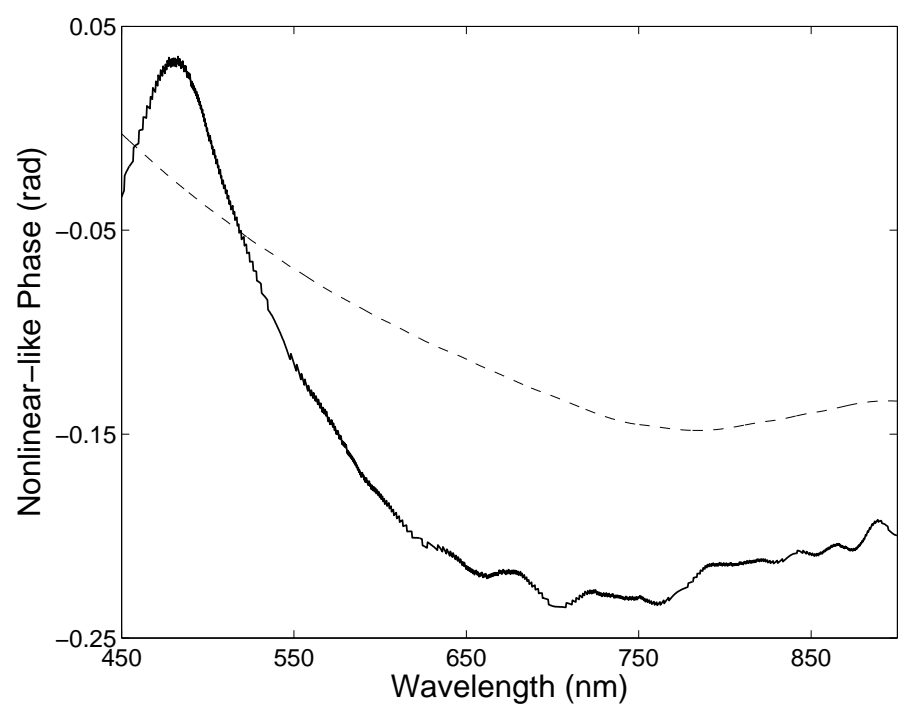

Fig. 4 The measured nonlinear-like phase as a function of wavelength for mirror 2 with that for aluminium (dashed)

of the $\mathrm{SiO}_{2}$ thin film on the silicon substrate. Figure 5 shows the comparison of the results of fitting the theoretical nonlinear-like phase $\delta(\lambda)$ to the measured one $\delta^{e}(\lambda)$ using the Levenberg-Marquardt least-squares algorithm [16]. The method determines the maximum-likelihood estimate of parameters $L$ and $d$ [11] that minimizes the figure-of-merit function $\chi^{2}$, defined by

$$
\chi^{2}(L, d)=\sum_{i=1}^{N}\left[\delta^{e}\left(\lambda_{i}\right)-\delta\left(\lambda_{i} ; L, d\right)\right]^{2},
$$

where $\lambda_{i}$ are wavelengths at which the fit was performed $(450$ to $900 \mathrm{~nm})$. Figure 5 demonstrates very good agreement between theory and experiment with the correlation coefficient as high as 0.99959 and the thin-film thickness $d=285.7 \mathrm{~nm}$.

We measured also the reflectance of the first sample of the $\mathrm{SiO}_{2}$ thin film on the silicon wafer by the threesteps procedure presented above. Figure 6 shows the comparison of the results obtained by fitting the theoretical reflectance $R(\lambda)$ to the measured one $R^{e}(\lambda)$ using the Levenberg-Marquardt least-squares algorithm. The method determines the maximum-likelihood estimate of the thin film thickness $d$ that minimizes the figure-ofmerit function $\chi^{2}$, defined in a similar way as that in Eq. (10). Figure 6 demonstrates very good agreement between theory and experiment with the correlation coefficient as high as 0.99904 and the thin-film thickness $d=285.3 \mathrm{~nm}$.

Figures 5 and 7 then show comparison of the experimental results with the theoretical ones concerning the nonlinear-like phase $\delta(\lambda)$ for the remaining three samples. Table 1 lists the corresponding thicknesses $d_{N L P}$ with the correlation coefficients $\mathrm{R}_{N L P}$. Similarly, Figs. 6 and 8 show the comparison of the experimental results with the theoretical ones concerning the reflectance $R(\lambda)$ 


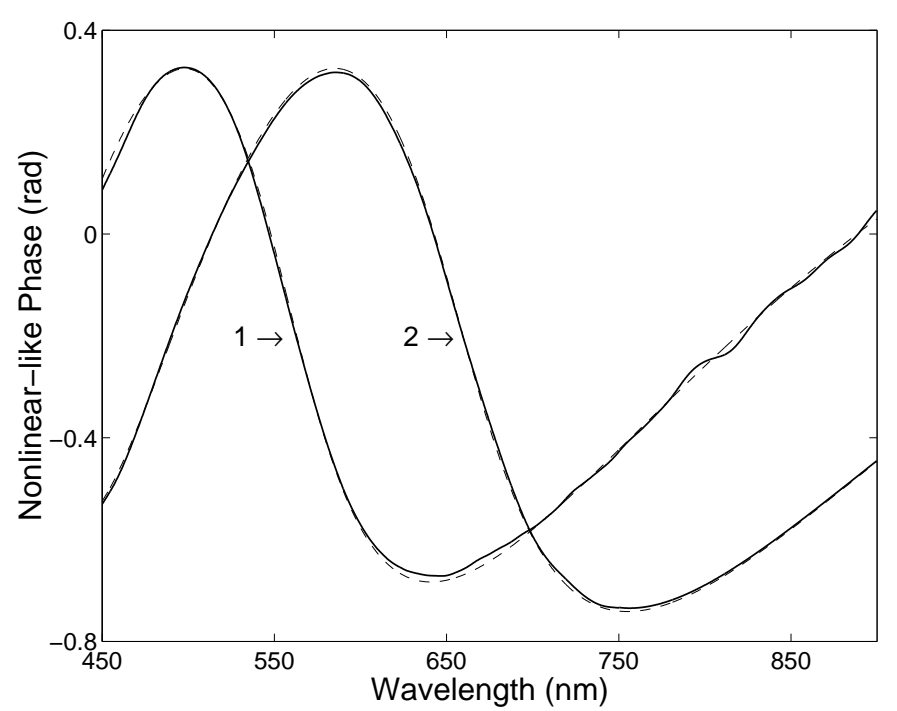

Fig. 5 The measured nonlinear-like phase as a function of wavelength with the corresponding fit (dashed) for samples 1 and 2

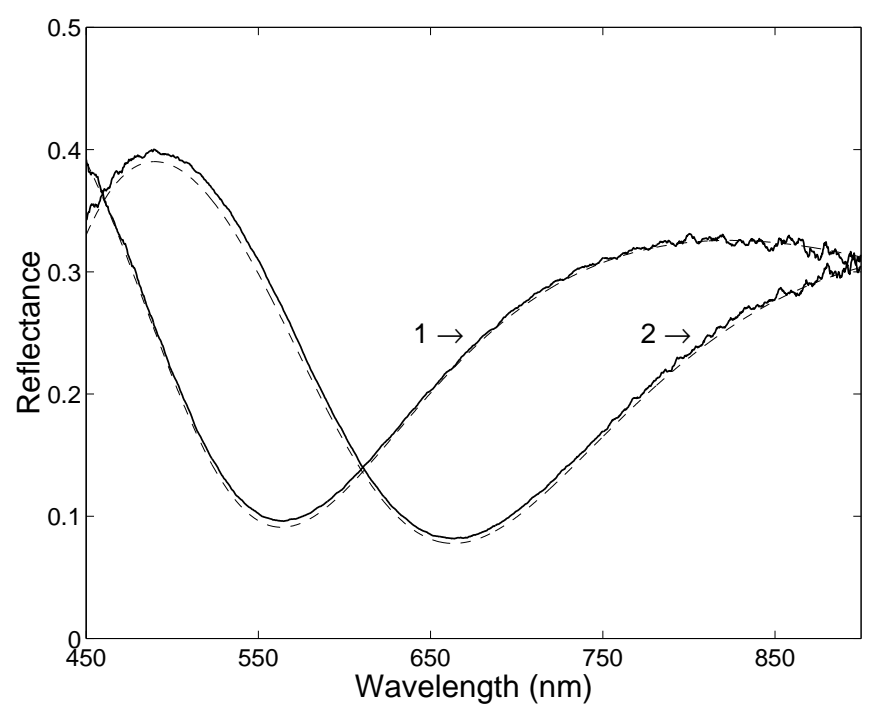

Fig. 6 The measured reflectance as a function of wavelength with the corresponding fit (dashed) for samples 1 and 2

and in Table 1 are listed the corresponding thicknesses $d_{R}$ with the correlation coefficients $\mathrm{R}_{R}$.

We can conclude from the obtained results that there is very good agreement between experiment and theory, especially in the case of the nonlinear-like phase. The correlation coefficients $\mathrm{R}_{N L P}$ indicate minimum discrepancy between the real structure and the theoretical model adopted. As can be deduced from the comparison of the results with those presented in a previous paper [12], the agreement is achieved by minimizing the systematic phase errors that are due to the optical components present in the interferometer. The correlation

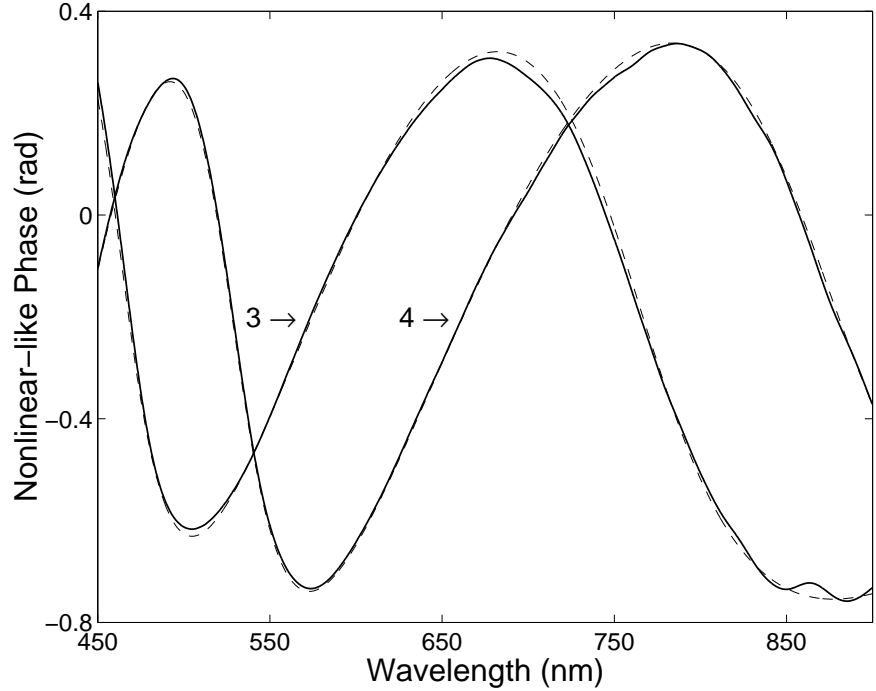

Fig. 7 The measured nonlinear-like phase as a function of wavelength with the corresponding fit (dashed) for samples 3 and 4

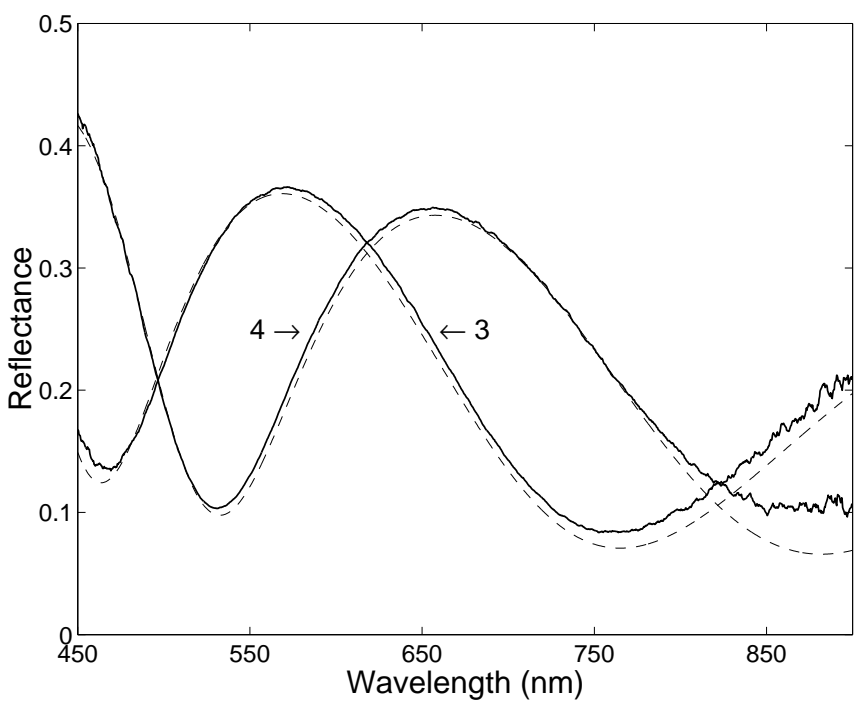

Fig. 8 The measured reflectance as a function of wavelength with the corresponding fit (dashed) for samples 3 and 4

coefficients $R_{R}$ show that the best agreement is reached for the first sample and the worst for the fourth one.

\section{Conclusions}

We used a new method for a precise measurement of the nonlinear-like phase of a thin-film structure. The method is based on white-light spectral interferometry and is combined with reflectometry. Phase errors due to optical elements of the interferometer are minimized by using the reference sample of known phase change on reflection. The feasibility of the method was confirmed in measuring the interferometric phase and reflectance 
Table 1 The oxidation time $T$ and the thicknesses $d_{R}$ and $d_{N L P}$ of the $\mathrm{SiO}_{2}$ thin films with the corresponding correlation coefficients $\mathrm{R}_{R}$ and $\mathrm{R}_{N L P}$

\begin{tabular}{cccccc}
\hline Sample No. & $T(\min )$ & $d_{R}(\mathrm{~nm})$ & $\mathrm{R}_{R}$ & $d_{N L P}(\mathrm{~nm})$ & $\mathrm{R}_{N L P}$ \\
\hline 1 & 122 & 285.3 & 0.99904 & 285.7 & 0.99959 \\
2 & 212 & 337.4 & 0.99804 & 336.8 & 0.99986 \\
3 & 326 & 390.2 & 0.99222 & 392.6 & 0.99916 \\
4 & 392 & 450.6 & 0.98664 & 449.2 & 0.99966 \\
\hline
\end{tabular}

of $\mathrm{SiO}_{2}$ thin film on a silicon wafer. The experimental data were used to determine the thin-film thickness for four samples provided that the optical constants for all the materials involved in the experiment are known. We confirmed very good agreement between the thicknesses obtained from the interferometric phase and reflectance measurements. An estimated uncertainty in determining the thicknesses from the measured nonlinear-like phase is better than $\pm 1 \mathrm{~nm}$ and the minimum thickness that can be measured by combination of white-light spectral interferometry and reflectometry is below $50 \mathrm{~nm}$ [7].

The results obtained serve as an illustration of the feasibility of a simple technique in measuring precisely the nonlinear-like phase, which is related to the phase change on reflection from a thin film. This method has the primary advantage in its normal incidence configuration over a technique such as ellipsometry. It can be combined with spectroscopic reflectometry and has potential applications in characterization of complex structures employing sophisticated parametric dispersion models and fitting of the measured functions, especially the nonlinearlike phase function. Both techniques can serve as a complement to spectroscopic ellipsometry.

Acknowledgements This research was partially supported by the Grant Agency of the Czech Republic (project No. 202/06/0531) and by the grant MSM6198910016.

\section{References}

1. R. A. Azzam, N. M. Bashara, Ellipsometry and Polarized Light (North-Holland, Amsterdam 1977)

2. G. E. Jellison, Jr., Thin Solid Films 290-291, 40 (1996)

3. T. M. Merklein, Appl. Opt. 29, 505 (1990)

4. M. Kildemo, V. Dalsrud, O. Fostad, Opt. Eng. 38, 1542 (1999)

5. S.-W. Kim, G.-H. Kim, Appl. Opt. 38, 5968 (1999)

6. U. Schnell, R. Dändliker, S. Gray, Opt. Lett. 21, 528 (1996)

7. Y.-S. Ghim, S.-W. Kim, Appl. Phys. Lett. 91, 091903 (2007)

8. T. Doi, K. Toyoda, Y. Tanimura, Appl. Opt. 36, 7157 (1997)

9. M.-C. Park, S.-W. Kim, Opt. Lett. 26, 420 (2001)

10. P. Hlubina, D. Ciprian, J. Luňáček, M. Lesňák, Appl. Phys. B 84, 511 (2006)
11. P. Hlubina, D. Ciprian, J. Luňáček, M. Lesňák, Opt. Express 14, 7678 (2006)

12. P. Hlubina, D. Ciprian, J. Luňáček, R. Chlebus, Appl. Phys. B 88, 397 (2007)

13. P. Hlubina, J. Luňáček, D. Ciprian, R. Chlebus, Opt. Commun. 281, 2349 (2008)

14. E. D. Palik Handbook of Optical Constants of Solids (Academic Press, Orlando 1995)

15. J. D. Plummer, M. D. Deal, P. B. Griffin: Silicon VLSI Technology Fundamentals, Practice and Modeling (Prentice Hall, Upper Saddle River 2000)

16. Optimalization Toolbox for Use with MATLAB (MathWorks, Mass. 2000) 